\title{
Bridging Recovery Initiative Despite Gaps in Entry (BRIDGE): study protocol for a randomized controlled trial of a bridge clinic compared with usual care for patients with opioid use disorder
}

\author{
David E. Marcovitz , Katie D. White, William Sullivan, Heather M. Limper, Mary Lynn Dear, Reagan Buie,
}

David A. Edwards, Cody Chastain, Kristopher A. Kast, Christopher J. Lindsell and Vanderbilt Learning Health System Investigators

\begin{abstract}
Background: Patients with substance use disorders are overrepresented among general hospital inpatients, and their admissions are associated with longer lengths of stay and increased readmission rates. Amid the national opioid crisis, increased attention has been given to the integration of addiction with routine medical care in order to better engage such patients and minimize fragmentation of care. General hospital addiction consultation services and transitional, hospital-based "bridge" clinics have emerged as potential solutions. We designed the Bridging Recovery Initiative Despite Gaps in Entry (BRIDGE) trial to determine if these clinics are superior to usual care for these patients.

Methods: This single-center, pragmatic, randomized controlled clinical trial is enrolling hospitalized patients with opioid use disorder (OUD) who are initiating medication for OUD (MOUD) in consultation with the addiction consult service. Patients are randomized for referral to a co-located, transitional, multidisciplinary bridge clinic or to usual care, with the assignment probability being determined by clinic capacity. The primary endpoint is hospital length of stay. Secondary endpoints include quality of life, linkage to care, self-reported buprenorphine or naltrexone fills, rate of known recurrent opioid use, readmission rates, and costs. Implementation endpoints include willingness to be referred to the bridge clinic, attendance rates among those referred, and reasons why patients were not eligible for referral. The main analysis will use an intent-to-treat approach with full covariate adjustment.

Discussion: This ongoing pragmatic trial will provide evidence on the effectiveness of proactive linkage to a bridge clinic intervention for hospitalized patients with OUD initiating evidence-based pharmacotherapy in consultation with the addiction consult service.
\end{abstract}

* Correspondence: david.marcovitz@vumc.org

Vanderbilt University Medical Center, Vanderbilt Psychiatric Hospital, 1603

23rd Ave South, Nashville, TN 37212, USA

(c) The Author(s). 2021 Open Access This article is licensed under a Creative Commons Attribution 4.0 International License, which permits use, sharing, adaptation, distribution and reproduction in any medium or format, as long as you give appropriate credit to the original author(s) and the source, provide a link to the Creative Commons licence, and indicate if changes were made. The images or other third party material in this article are included in the article's Creative Commons licence, unless indicated otherwise in a credit line to the material. If material is not included in the article's Creative Commons licence and your intended use is not permitted by statutory regulation or exceeds the permitted use, you will need to obtain permission directly from the copyright holder. To view a copy of this licence, visit http://creativecommons.org/licenses/by/4.0/ The Creative Commons Public Domain Dedication waiver (http://creativecommons.org/publicdomain/zero/1.0/) applies to the data made available in this article, unless otherwise stated in a credit line to the data. 
Trial registration: ClinicalTrials.gov NCT04084392. Registered on 10 September 2019. The study has been approved by the Vanderbilt Institutional Review Board. The current approved protocol is dated version May 12, 2021.

Keywords: Pragmatic clinical trial, Substance use disorder, Opioid use disorder, Bridge clinic

\section{Background}

Patients with substance use disorders (SUDs) are overrepresented among general hospital inpatients [1], and their admissions are associated with longer lengths of stay and increased readmission rates $[2,3]$. There is also broad understanding that the majority of patients with SUD do not seek care, falling into the "treatment gap" on epidemiological surveys [4]. The national opioid crisis has brought renewed attention to the question of how to most effectively reach patients struggling with opioid use disorder (OUD) as early as possible in their course of illness in order to reduce morbidity and mortality $[5,6]$.

Treatment with medications for OUD (MOUD) is known to be effective. Patients treated with MOUD have higher rates of retention in treatment, lower rates of illicit drug use, fewer infectious complications, and reduced opioid overdose deaths than those who do not receive MOUD [13, 14]. Rates of sustained abstinence are less than 10\% for patients not treated with MOUD, such as buprenorphine-naloxone [10-12]. However, when persons with OUD engage with the medical system, the availability of evidence-based treatment, including MOUD, is limited [7]. One study reported that less than $8 \%$ of patients with injection drug use-related endocarditis were initiated and referred for outpatient treatment including MOUD [8, 9]. The combination of high utilization of medical services and poor access to evidence-based addiction treatment has heightened awareness of the need to integrate addiction treatment into routine medical care.

Efforts focused on improving access to addiction care have historically been divided into inpatient and outpatient efforts, with a more recent focus on the transitions of care between these two settings. Preceding the opioid crisis, efforts to integrate SUD treatment into primary care were underway [15], and more recently, efforts have focused on collaborative care for OUD in the primary care setting as a means to increase the availability of MOUD [16]. For hospitalized patients, general hospital addiction consultation services have now been described at multiple institutions $[6,17,18]$ with some studies showing an impact on 30-day abstinence, addiction severity, and readmission [17, 19, 20]. In order to better support such inpatient efforts and link patients into longitudinal care more rapidly, transitional "bridge" clinics have emerged as potential solutions to the problems of care continuum fragmentation, increased length of stay, and high readmission rates [21]. Though these bridge clinics vary in many respects, common features include their transitional nature, low threshold and low wait-time to access, and geographic co-location within the hospital system to which the patient presented.

Though the potential benefits of bridge clinics are many, little is known about the effectiveness of the bridge clinic model. At the patient level, it remains to be seen whether the intervention can effectively retain patients, reduce relapse to illicit substances, and improve medical outcomes alongside quality of life. At the system level, the potential benefits of the bridge clinic model are promising but to date unproven.

One area of potential for significant impact involves hospital admissions for patients with infectious complications of injection drug use. These admissions have been increasing year-over-year [22], with one study in North Carolina estimating a cost per admission of $\$ 50,000$ for endocarditis (with $42 \%$ uninsured or on Medicaid) [23]. Another study involving a safety net hospital described an annual cost of $\$ 11.4$ million to care for 349 patients with infectious complications of intravenous drug use, in this case with a $90 \%$ rate of uninsured or Medicaid status [24]. These national trends were reflected in our own institutional data with a 55\% increase in admissions with OUD-related diagnostic codes in the first 6 months of 2018 compared to the year prior. The average length of stay for patients with OUD and need for intravenous antibiotic treatment was 15 days compared to a hospital-wide average of just 3 days. Whether the use of a bridge clinic model improves patient outcomes while reducing resource use remains unknown.

\section{Objectives}

We designed the Bridging Recovery Initiative Despite Gaps in Entry (BRIDGE) trial to test the hypothesis that patients assigned to a bridge clinic intervention will have reduced hospital LOS, reduced readmission rates, improved linkage to care, improved MOUD fill rates, fewer relapses, improved quality of life, and reduced total cost of care compared with patients assigned to usual care.

\section{Methods and design Methods}

The bridge clinic was not designed as a research intervention; rather, this prospective study was designed to evaluate the effectiveness of the clinic as it was being 
implemented as a part of clinical care in the study setting. The clinic was initially deployed in order to address the unpredictable and fragile transitions for patients with SUD leaving the adult hospital and emergency department (ED), including those with injection-related infections. The model is predicated on the theory that both SUD and the co-occurring disorders that accompany it disrupt the reward, stress, and executive planning neurocircuitry such that transitions in care must be facilitated to improve retention and engagement in care [25]. The deployment of this new model of care provided an opportunity for rigorous evaluation of its effectiveness given that the clinic, founded with limited hospital resources, has a fixed capacity and therefore those patients for whom referral was not possible can serve as a control group. The effectiveness of this new model of care must be evaluated given funding sources are limited and leveraged to support programs that demonstrate improved outcomes. Moreover, evaluation of both the clinical efficacy and potential financial savings of the bridge clinic model will determine the utility of expansion. This manuscript has been written in accordance with Standard Protocol Items: Recommendations for Interventional Trials (SPIRIT) guidelines, shown in more detail in an additional file (see Additional file 1) [26].

\section{Study design}

The BRIDGE trial is a pragmatic, single-center, superiority, randomized, controlled trial beginning 25 November 2019 at Vanderbilt University Hospital in Nashville, TN, USA. As this is a pragmatic trial testing real-world effectiveness, no additional strategies were included to improve adherence and there were no restrictions on concomitant care or other interventions.

\section{Study sites and period}

Vanderbilt University Hospital is an $\sim 800$ bed, tertiary care adult hospital. The study commenced on 25 November 2019 and is anticipated to be completed in 18 months.

\section{Population}

The population included in this study are patients admitted for OUD and being considered for MOUD in consultation with the addiction consult service. The inclusion and exclusion criteria are:

\section{Inclusion criteria:}

- Aged $\geq 18$ years

- Active OUD being considered for MOUD evidenced by accepting a transitional prescription for buprenorphine-naloxone or intramuscular naltrexone injection
- Outpatient plans not fixed prior to admission, defined as a patient reporting a prior relationship with an MOUD provider that they intend to continue after discharge, including cash pay practices

- Patient lives in one of the counties that comprise Middle Tennessee, shown in an additional file listing appropriate zip codes (see Additional file 2), in order to ensure a reasonable chance of the patient having regular transportation to the clinic

\section{Exclusion criteria:}

- Severe, active co-occurring psychiatric disorders that require a higher level of psychiatric care

- Patients for whom methadone maintenance is deemed the best choice of MOUD

- Patients previously randomized in this study

- Patients who previously were referred to the bridge clinic prior to study initiation

\section{Enrollment and randomization}

In the hospital setting, the addiction consult service is notified of potentially eligible patients via an electronic consult order or referral from the general psychiatry service. The addiction consult service is comprised of an addiction psychiatrist, a psychiatric nurse practitioner, a social worker, a nurse case manager, and a recovery coach, with additional fellows and residents rotating through with some regularity. Those patients for whom an order for an addiction consultation is placed are evaluated by one of the psychiatric providers for MOUD. The addiction consult service social worker further screens the patient for inclusion. Once inclusion eligibility is met, the social worker proceeds with randomization via REDCap.

The capacity of the bridge clinic is fixed-the physical clinic occurs all day Friday and we are unable to see more than a set number of new and follow-up patients-so volume is driven by the number of patients referred from the general adult hospital, the adult emergency department, and the psychiatric emergency department. We allocated a set number of intakes per week to emergency services, but only those patients being referred from the general adult hospital are eligible for randomization and enrollment. Due to capacity limitations, the clinic is unable to care for all patients that could be referred. We are leveraging this gap between demand and capacity to use randomization to determine which patients are offered referral from the adult hospital; randomization ensures that selection is unbiased and allows a meaningful comparison of outcomes between those referred and those not referred. Patients deemed eligible for MOUD are randomized to being 
offered referral to the bridge clinic or to usual care with referral to community MOUD providers. Since patients are enrolled under a waiver of informed consent, the option of declining the trial is not available. Patients can, however, decline the bridge clinic if it is made available to them. During the duration of the trial itself, the bridge clinic is not available outside of the auspices of the study for those persons who were study eligible.

Randomization occurs using a pre-specified sequence deployed using REDCap's randomization module [27]. Randomization proceeds regardless of whether the patient prefers to attend a higher level of care (e.g., intensive outpatient, partial hospital program, or residential rehabilitation) on the assumption that these patients will want an outpatient MOUD program after completion of such care. Given the capacity of the clinic and the number of patients eligible, the randomization ratio is set to $1: 1$, and this is evaluated bi-weekly to maintain the bridge clinic at full capacity. We chose not to stratify randomization based on medical severity because we were unsure a priori how to define severity levels that would meaningfully impact the primary and secondary outcomes. Because this study involves only observational data collection after randomization to bridge clinic or no bridge clinic availability, there are no plans to withdraw participants or discontinue them in the study. A schedule of events is shown in Fig. 1.

\section{Intervention delivery}

The Vanderbilt bridge clinic model includes a team that provides wraparound services for patients with SUD during their post-acute care, regardless of insurance status. The pharmacy provides institutionally grant-funded medication to uninsured patients, and charges (including visit and lab charges) for these patients are written off by the institution. As of September 3, 2020, 57\% of the clinic patients were uninsured. The care team is comprised of four different physician specialists (addiction psychiatry, internal medicine, infectious diseases, and pain-anesthesia), a psychiatric nurse practitioner, two licensed social workers, a nurse case manager, and two recovery coaches. All non-medical team members have some background or additional training in the management of addiction and other mental health conditions. These non-medical providers collectively provide tailored case management and other brief psychotherapeutic interventions (e.g., motivational interviewing, education, twelve-step facilitation).

Team members work across addiction treatment settings at our institution, supporting the addiction consult service, the ED, and longitudinal outpatient clinics. The addiction consult service initiates care with patients in the hospital and, for those referred to the bridge clinic, they maintain contact thereafter both remotely and through in-person clinic visits. Patients generally present in-person, though there has been a transition to telemedicine for some patients for brief periods during the COVID-19 pandemic.

Our bridge clinic opened on May 17, 2019, with service one-half day each week in two different locations: one for primary care, psychiatry, and pain-anesthesia providers on Friday mornings, and one for infectious diseases on Friday afternoons. On November 8, 2019, the clinic was relocated to a space that accommodates the entire team all-day Friday, facilitating a traditional medical model in which a medical assistant situates patients in one of seven rooms after collecting intake information, where other providers rotate seeing the patient as needed. This improves continuity of care as it allows multiple providers to see a patient together when there is a need for care coordination.

The bridge clinic primarily cares for patients with OUD who may have any number of co-occurring psychiatric, SUD, and medical issues. Patients are asked to present weekly to the clinic for the first 8 weeks of treatment (assuming they maintain abstinence), then twice monthly. The target period for stabilization and transition to a long-term treatment program is no more than 3 months, though in practice this may be as long as a year if there are challenges to successful transition. We estimate based on early anecdotal experience with the clinic referral process that $75 \%$ of new patients scheduled will attend, and those patients will attend on average for eight out of ten visits over the initial 12-week period.

The visit model and provider-patient pairing are designed to be tailored to patient needs within this general framework. At the time of referral into the clinic, the addiction consult service social worker and nurse case manager prioritize pairing patients with a medical provider based on the patient's co-occurring medical and psychiatric conditions. Because of the flexibility of the model, a medical provider can obtain additional evaluations from other specialty providers in the clinic (e.g., psychiatry provider asking the primary care provider to assess a rash, or infectious diseases provider asking painanesthesia to evaluate low back pain) either same-day or at a subsequent visit. Social workers, case managers, and recovery coaches also adjust their pairing over time with patients, though we try to have a social worker and recovery coach follow each patient longitudinally. Medical and non-medical providers use huddles before or after visits to discuss treatment plans for patients, especially patients whose recovery is tenuous and may require referral to a higher level of care. Social work, case management, and recovery coaching staff facilitate referrals to other levels of care and communicate with patients outside of the physical clinic to advance these treatment 


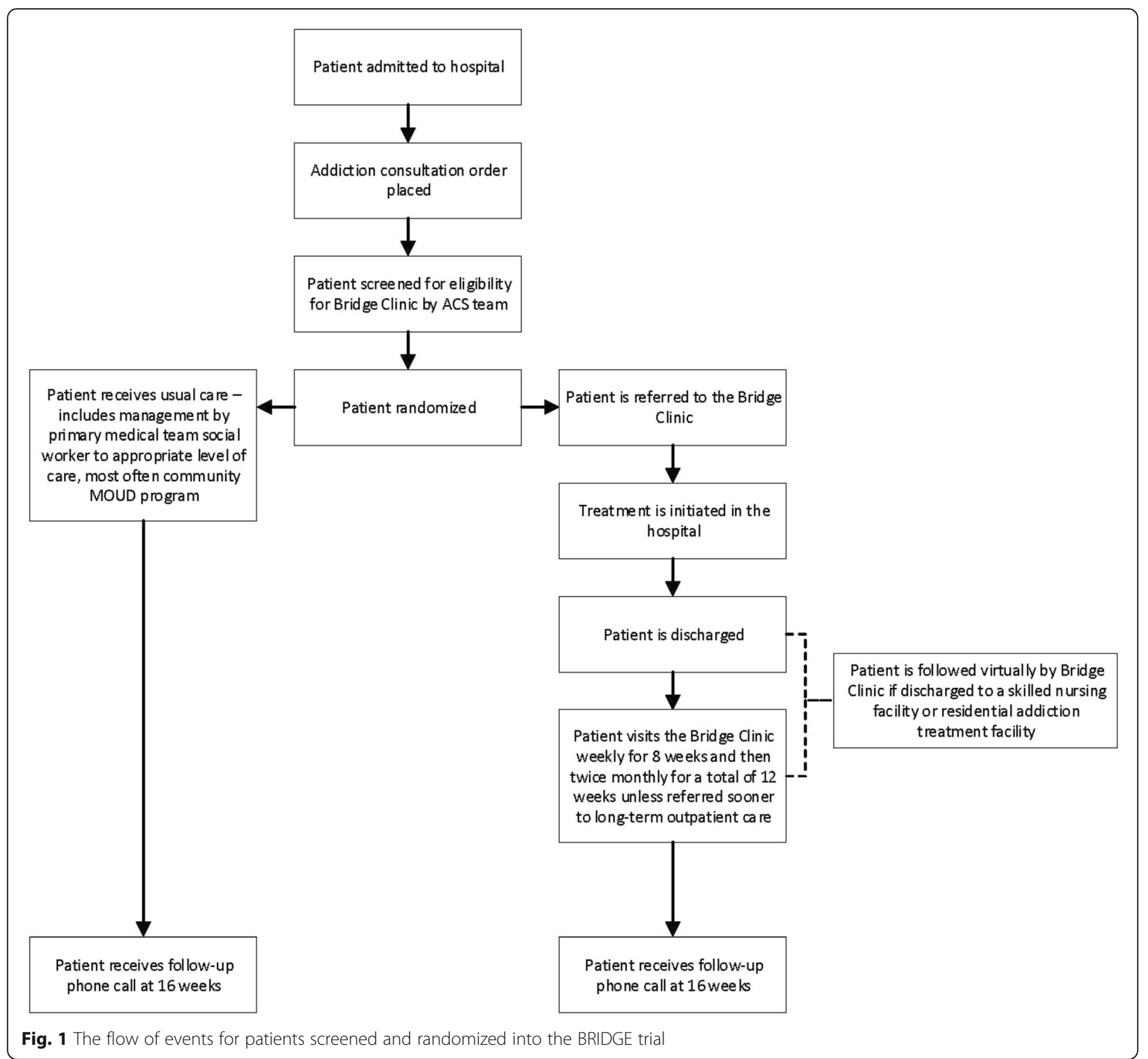

plans. Permanent transition out of the clinic into appropriate long-term care is addressed as often as every visit, whether the patient requires a higher level of care or not.

Patients are seen for each visit by at least one medical provider and at least one member of the team who can provide psychotherapy, peer support, or case management until they demonstrate 8 weeks of abstinence. The patient provides a urine specimen for toxicology testing at each visit. In rare cases, patients will opt to leave a visit before seeing a non-medical provider, but this is discouraged. After patients have finished seeing the appropriate providers, they are directed from the clinic to the lab and pharmacy. The lab is in the same building and the institutional pharmacy is located just two blocks away. Point-of-care urine drug screening is not currently done but is being developed for implementation along with another planned relocation to a custom-designed facility in 2021. Insured patients can opt to fill medications at an external pharmacy, although this is rare. Prescriptions for controlled substances (e.g., buprenorphine, gabapentin) are refilled at each visit, with 7-day supplies during initial encounters. Prescription-monitoring program entries are reviewed for each patient at each visit.

Usual care is the traditional care provided by the inpatient medical team's assigned social worker. Patients randomized to usual care are informed by the addiction consult service social workers that the social worker for their primary medical team will be in communication with them about outpatient resources for MOUD and 
recovery support. An electronic pass-off is completed by the addiction consult service social worker to the primary team social worker, and this pass-off is also documented in the medical record. The primary medical team social worker then meets with the patient for additional assessment and makes referrals appropriate to the individual patient's transportation and insurance needs. The addiction consult service continues to work with the patient to initiate MOUD and address comorbidity during their hospital admission.

\section{Data collection}

Patient information is documented as a component of usual clinical care and will be extracted from the electronic health record (EHR) for this study. This includes demographic information (i.e., age, gender, race, ethnicity, socio-economic indicators) as well as the length of stay.

Many patients are admitted with infections related to injection drug use and require weeks of treatment with intravenous antibiotics. As part of the BRIDGE trial, we have developed a protocol to assess candidacy for outpatient parenteral antibiotics therapy (OPAT) for patients who are randomized to the bridge clinic for postacute follow-up care. Information obtained during OPAT candidacy screening is entered into a secure data management system, REDCap, including the history of prior hospital misuse, housing status, telephone access, 24-7 access to a refrigerator for antibiotics storage, presence or absence of SUD in cohabitants, willingness to allow home health services into the home, presence or absence of home support person, and willingness of home support person to confirm the presence of stable housing, to assist with antibiotic administration, and to assist patient with attending medical appointments. These data inform the eligibility of the patient for the OPAT protocol.

Prior to April 2021, only patients randomized to the bridge clinic were potentially eligible for OPAT. In theory, this would favor the bridge clinic in terms of the primary outcome (LOS), though this is not a limitation per se but rather part of the central hypothesis. In April 2021, in response to extreme hospital bed demand related to COVID-19, we adjusted our protocol such that OPAT eligibility took precedence over randomization. At that point, any patient deemed eligible for OPAT would be referred to bridge clinic and not included in the trial.

Part of usual care for patients seen by the addiction consult service and initiated on MOUD also includes a 16week follow-up phone call, at which time additional information is collected. This call is also conducted for patients randomized to attend the bridge clinic. These data include initial attendance at outpatient MOUD follow-up, self- reported relapse to use of illicit opioids and frequency of use in preceding 30 days, number of overdoses since discharge, cross-over from usual care to bridge clinic or vice versa, and a measure of overall psychological well-being (Schwartz Outcome Scale-10) [28-30].

At the time of the 16-week follow-up, case managers make up to three telephone calls, leaving voicemails if the patient does not respond. Beginning in August 2020 and due to an initially low response rate, each call is accompanied by a text message from recovery coaches on their institutional mobile device or through our institutional patient portal for those patients who prefer it. Case managers attempt to engage additional contacts in the medical record when patients are unable to be reached. Finally, some patients cannot be reached for documented reasons at 16 weeks, such as incarceration or death.

\section{Primary outcomes}

The primary outcome measured is the overall index hospital admission length of stay. Hospital length of stay is an accepted metric in operational health care research. It was chosen in this case in light of the observation that patients with OUD were often mismatched with the treatment setting for two reasons: (1) they were often being kept in the hospital due to a standard of care holding that patients with OUD should not be eligible for ambulatory parenteral antibiotic therapy given the risk of misuse of central venous access devices, despite evidence that this can be done safely [31, 32], and (2) the general medical hospital does not tend to provide a therapeutic environment for patients in early recovery from OUD who are isolated and lack access to behavioral treatments and community mutual help supports. Therefore, reduction in hospital LOS represents an easily measured proxy for improving an outcome that matters to both providers and patients in terms of advancing OUD recovery and providing value to the hospital system.

\section{Secondary outcomes}

The secondary outcomes, all assessed for the 16-week follow-up period, are:

- Linkage to MOUD provider, defined as attending at least one visit with a MOUD provider after discharge, based on self-report at 16-week follow-up call. Such engagement is considered a process measure in the field for engagement correlated with outcome measures related to abstinence at 6 months follow-up [33].

- Self-reported buprenorphine-naloxone (or naltrexone) prescriptions filled, a related process measure

- Readmission rates 
- ED visits

- Hospital-free days

- ED-free days

- Recurrent opioid use

- Overall quality of life as measured by the Schwartz Outcome Scale-10 (SOS10) and EQ-5D-5L. We chose to add these additional quality measures given that the OUD literature has often neglected socalled functional outcomes that matter to patients other than abstinence from illicit substances, though certainly abstinence is thought to correlate with improved quality of life [34].

- Overdose

- Mortality

- Cost of care, including total costs for each admission and care resources utilized. Our medical center finance team is able to provide these costs as another useful measure of value provided by the intervention which may capture LOS reduction but also reduction in other costs that the bridge clinic may influence like readmission, substance misuse in the hospital with reinfection, and patients leaving the hospital against medical advice. We will not be able to easily capture costs associated with the use of other medical centers in the region for purposes of this study.

Additional outcomes for patients with infection suitable for outpatient management include:

- New, persistent, or recurrent infection (as defined by a positive culture and/or change in antibiotic regimen)

- Completion of antibiotic therapy

- The number of days from negative blood culture to first hospital discharge

Implementation measurements include acceptance of referral to bridge clinic and cross-over from usual care to bridge clinic and vice versa, as well as any reasons for ineligibility.

\section{Analysis plan \\ Statistical analysis}

Initially, we will characterize participants overall and grouped by study arm using means with standard deviations, medians with interquartile range, and counts with percentages as appropriate. The primary analysis will compare the length of index hospital stay between those offered referral to the bridge clinic and those not offered referral to the bridge clinic on an intent-to-treat basis. We will use a generalized linear model with group assignment as the primary predictor variable, with adjustment for important covariates. Multiple imputation will be used for missing covariates; length of stay will not be missing for any cases. We expect to model length of stay as a continuous outcome. We may choose a proportional hazards model or a gamma generalized linear model with a log-link function if the data are substantially skewed; we do not expect this based on our experience with the length of stay in this patient cohort. We have one primary outcome, and we will use a critical $p$-value of 0.05 to test our primary hypothesis.

Secondary and exploratory analyses will also use the intent-to-treat analysis set. Binary outcomes will be compared between groups using logistic regression, adjusted for covariates. Ordinal outcomes will use a proportional odds model. Costs are expected to be skewed and so a proportional hazards model or a gamma generalized linear model with a log-link function will be chosen.

\section{Power calculation}

The mean length of stay for patients meeting the inclusion criterion in the 12-to-18-month period before bridge clinic was established was 15 days, ranging from 3 to 42 with a standard deviation of about 15 days. With 358 patients per study arm, we would have $90 \%$ power to detect a reduction in length of stay of 3 days, assuming the common standard deviation was decreased to 12.5 days. If the common standard deviation only reduced to 14 days, we would still have $80 \%$ power to detect a difference. We therefore selected a sample size of 700 , with 350 randomized to each arm. We expected to complete this study within 18 months.

Because of the disruption caused by COVID-19 and lower than expected enrollment, midway through the recruitment period, we re-estimated the sample size based on the experience of patients. Blinded to allocation, we estimated the distribution of length of stay for all enrolled patients. We found the mean length of stay was shorter, at 9 days, with a standard deviation of 11. The length of stay was also decidedly right skewed. Assuming a reduction in length of stay of 1.5 days is meaningful, about 168 patients per group would be required to have $80 \%$ power to detect a difference. Given our current accrual rate of four patients per week, if we continue enrolling for 12 months as originally planned, we will obtain a sample size of 336, which is sufficient to have more than $80 \%$ power for our primary outcome.

\section{Data confidentiality, sources, and sharing}

Data for this trial will be extracted from the electronic health record and ancillary systems as all information is generated in the usual process of care. Data will be entered into REDCap, which provides a secure environment for the maintenance of the data. Analytical datasets will be stored on secure servers without direct 
identifiers. On completion of this study, data will be made available on submission of a methodologically sound proposal that is accompanied with appropriate regulatory approvals.

\section{Presentation of the results}

After completion of enrollment and data analysis, the results of the trial will be communicated through manuscript publication. Submission will include public access to the full study protocol and statistical code. Authorship will be based on the International Committee of Medical Journal Editors guidelines (2018), and professional writers will not be used. The results will also be presented at local and national conferences, posted on ClinicalTrials.gov, and used to inform local evidencebased practice committees on care options for hospitalized patients with OUD.

\section{Discussion}

Upon completion, the BRIDGE trial will provide the most comprehensive data to date on the effect of a multispecialty, multidisciplinary transitional clinic focused on continuity of access to MOUD initiated by an addiction consult service during acute care in a general hospital.

Several potential threats to the validity of the trial exist. Because data are being obtained based on charting by practicing clinicians in the electronic health record (EHR), there may be missing data. For the primary outcome, there will be many factors other than the bridge clinic intervention that may impact the length of stay. We expect randomization to mitigate the risk that one arm was disproportionately affected by such factors. Given that all providers are aware of the trial itself and the randomization status of a given patient, it is possible that unconscious or conscious biases will lead to differential treatment of patients based on randomization condition in ways that go beyond the scope of the trial itself. Furthermore, all patients considered for the trial receive addiction consult services, an intervention which may reduce observed effect size by reducing the length of stay compared to hospitalized patients with OUD who do not receive addiction consult services.

Our study is a pragmatic trial, which provides the opportunity to evaluate the effectiveness of an intervention in real-life routine practice conditions [35]. Inherent in this design however is the potential for a lack of external validity. Additionally, with a single-center trial, there is limited ability to generalize the study results. Availability of an addiction consult service is not universally available or feasible in all general hospital contexts.

Other than certain objective measures such as ED visits, readmissions, and costs of care, many secondary outcomes are based on self-report by patients at their 16-week follow-up. There is a high risk of bias as to which patients staff reached to obtain 16-week follow-up data. For example, those that are more successful with their recovery may be more likely to maintain continuous cell phone access and to want to report on their progress. If considerable bias in follow-up rates is observed, we may test the external validity of our findings by repeating our primary analysis using inverse probability weighted for propensity to follow-up.

Additional sources of bias include changes in the availability of community-based care and the ability of all patients to engage in community-based mutual help and other supports as this trial coincided with the COVID19 pandemic. Many of these societal changes could have affected both randomization groups equally, though a reduction in community MOUD programs as well as transportation and other resources could have disproportionately affected patients in the usual care group. Finally, we did consider the potential influence of patients departing the hospital against medical advice (AMA) in the design and determined that it would likely bias the trial results towards the null. Patients with longer lengths of stay may be more likely to leave AMA and thus reduce the LOS overall. If our hypothesis is supported, this would have a greater influence on the TAU arm when compared with the BRIDGE arm.

Despite the challenges and potential biases, the ongoing pragmatic BRIDGE trial will provide evidence on the effectiveness of proactive linkage to a bridge clinic intervention for hospitalized patients with OUD initiating evidence-based pharmacotherapy in consultation with the addiction consult service.

\section{Trial status}

The status of this trial is currently ongoing with enrollment aimed to be completed in the fall of 2021 .

\section{Abbreviations}

EHR: Electronic health record; ED: Emergency department; MOUD: Medication for opioid use disorder; OUD: Opioid use disorder; OPAT: Outpatient parenteral antibiotics therapy; SOS-10: Schwartz Outcome Scale-10; SPIRIT: Standard Protocol Items: Recommendations for Interventional Trials; SUD: Substance use disorder

\section{Supplementary Information}

The online version contains supplementary material available at https://doi. org/10.1186/s13063-021-05698-4.

Additional file 1. Standard Protocol Items: Recommendations for Interventional Trials (SPIRIT) guidelines, PDF, completed checklist of SPIRIT guidelines

Additional file 2. Zip Codes for Inclusion, PDF, complete list of all zip codes of residency considered for inclusion in enrollment

\section{Acknowledgements}

The trial is being conducted within the Vanderbilt Learning Healthcare System. The authors thank the patients and the clinical care teams at 
Vanderbilt University Medical Center for making this study possible. The Vanderbilt Learning Health System Investigators study group is comprised of Gordon Bernard, Bob Dittus, Cheryl Gatto, Frank Harrell, Paul Harris, Tina Hartert, Cathy Ivory, Jim Hayman, Kevin Johnson, Ruth Kleinpell, Sunil Kripalani, Patrick Luther, Mariann Piano, Jill Pulley, Todd Rice, Russell Rothman, Matt Semler, Shon Dwyer, Robin Steaban, Thomas Nantais, Philip Walker, Asli Weitkamp, Consuelo Wilkins, Adam Wright, and Autumn Zuckerman. The Vanderbilt Learning Healthcare System Investigators study group provides generalized oversight for project development, ensures alignment with institutional priority, attempts to maximize pragmatism, and offers tailored regulatory guidance.

\section{Ethics and dissemination}

The study was approved by the local Institutional Review Board. Results will be submitted for publication, presented at national conferences, and will guide local practice decisions.

\section{Authors' contributions}

Each author has made substantial contributions to the conception, design, or implementation of the work. DM, KW, CC, WS, MLD, RB, and CL were involved in the conception and design of the work; DM, KW, CC, WS, DE, and KK were involved in implementation; DM led the manuscript writing and revision work. All authors have provided critical input to the manuscript and have read and approved the submitted version.

\section{Authors' information}

BRIDGE investigators Vanderbilt University Medical Center, Nashville, TN, USA: David Marcovitz, Katie White, Cody Chastain, William Sullivan, Reagan Buie, Mary Lynn Dear, David Edwards, Heather Limper, Kristopher Kast, and Christopher J Lindsell for the Vanderbilt Learning Healthcare System Investigators.

\section{Funding}

The project described was supported by the Vanderbilt Institute for Clinical and Translational Research (VICTR) Learning Healthcare System Platform under CTSA award No. UL1 TR002243 from the National Center for Advancing Translational Sciences. Its contents are solely the responsibility of the authors and do not necessarily represent official views of the National Center for Advancing Translational Sciences or the National Institutes of Health.

\section{Availability of data and materials}

On completion of the study, investigators external to the research team may request to collaborate on secondary analyses. With appropriate IRB approval and data use agreements in place, de-identified datasets may be released on reasonable request. All statistical code will be made publicly available with the analysis. The investigators plan to publish trial results without assistance from outside professional writers. The investigators have no publication restrictions. Materials that are shared with patients as part of the bridge clinic program are under continuous development and are available from the corresponding author upon request.

\section{Declarations}

\section{Ethics approval and consent to participate}

The trial was reviewed and approved by the Institutional Review Board (IRB) with a waiver of informed consent. IRB approval number 191269.

\section{Consent for publication}

Not applicable.

\section{Competing interests}

The authors declare that they have no competing interests.

Received: 19 March 2021 Accepted: 8 October 2021

Published online: 30 October 2021

\section{References}

1. Laine C, Hauck WW, Gourevitch MN, Rothman J, Cohen A, Turner BJ. Regular outpatient medical and drug abuse care and subsequent hospitalization of persons who use illicit drugs. Jama. 2001;285(18):2355-62. https://doi.org/10.1001/jama.285.18.2355.

2. Mark T, Tomic KS, Kowlessar N, Chu BC, Vandivort-Warren R, Smith S. Hospital readmission among Medicaid patients with an index hospitalization for mental and/or substance use disorder. J Behav Health Serv Res. 2013;40(2):207-21. https://doi.org/10.1007/s11414-013-9323-5.

3. Gili-Miner M, Béjar-Prado L, Gili-Ortiz E, Ramírez-Ramírez G, López-Méndez J, López-Millán J-M, et al. Alcohol use disorders among surgical patients: unplanned 30-days readmissions, length of hospital stay, excessive costs and mortality. Drug Alcohol Depend. 2014;137:55-61. https://doi.org/10.101 6/j.drugalcdep.2014.01.009.

4. Abuse S, Administration MHS. Results from the 2013 National Survey on Drug Use and Health: summary of national findings. NSDUH Ser H-48 HHS Publ NoSMA14-4863. 2014:1-143.

5. Weimer M, Morford K, Donroe J. Treatment of opioid use disorder in the acute hospital setting: a critical review of the literature (2014-2019). Curr Addict Rep. 2019;6(4):339-54. https://doi.org/10.1007/s40429-019-00267-x.

6. Marcovitz D, Nisavic M, Bearnot B. Staffing an addiction consult service: Psychiatrists, internists, or both? In: Staffing an addiction consult service: psychiatrists, internists, or both? 2019.

7. Abraham AJ, Andrews CM, Harris SJ, Friedmann PD. Availability of medications for the treatment of alcohol and opioid use disorder in the USA. Neurotherapeutics. 2020;17(1):1-15. https://doi.org/10.1007/s13311-01 9-00814-4.

8. Rosenthal ES, Karchmer AW, Theisen-Toupal J, Castillo RA, Rowley CF. Suboptimal addiction interventions for patients hospitalized with injection drug use-associated infective endocarditis. Am J Med. 2016;129(5):481-5. https://doi.org/10.1016/j.amjmed.2015.09.024.

9. Mihm AE, Cash MC, Nisly SA, Davis KA. Increased awareness needed for inpatient substance use disorder treatment in drug use-associated infective endocarditis. J Gen Intern Med. 2020;35(7):1-3. https://doi.org/10.1007/s11 606-019-05607-1.

10. Fudala PJ, Bridge TP, Herbert S, Williford WO, Chiang CN, Jones K, et al. Office-based treatment of opiate addiction with a sublingual-tablet formulation of buprenorphine and naloxone. N Engl J Med. 2003;349(10): 949-58. https://doi.org/10.1056/NEJMoa022164.

11. Kakko J, Svanborg KD, Kreek MJ, Heilig M. 1-year retention and social function after buprenorphine-assisted relapse prevention treatment for heroin dependence in Sweden: a randomised, placebo-controlled trial. The Lancet. 2003;361(9358):662-8. https://doi.org/10.1016/S0140-6736(03)12 600-1.

12. Weiss RD, Rao V. The prescription opioid addiction treatment study: what have we learned. Drug Alcohol Depend. 2017;173:S48-54. https://doi.org/1 0.1016/j.drugalcdep.2016.12.001.

13. Mattick RP, Breen C, Kimber J, Davoli M. Buprenorphine maintenance versus placebo or methadone maintenance for opioid dependence. Cochrane Database Syst Rev. 2014;2. https://doi.org/10.1002/14651858.CD002207.pub4

14. Connery HS. Medication-assisted treatment of opioid use disorder: review of the evidence and future directions. Harv Rev Psychiatry. 2015;23(2):63-75. https://doi.org/10.1097/HRP.0000000000000075.

15. Saitz R. Screening and brief intervention enter their 5 th decade. Subst Abuse. 2007;28(3):3-6. https://doi.org/10.1300/J465v28n03_02.

16. Watkins KE, Ober AJ, Lamp K, Lind M, Setodji C, Osilla KC, et al. Collaborative care for opioid and alcohol use disorders in primary care: the SUMMIT randomized clinical trial. JAMA Intern Med. 2017 Oct 1;177(10):1480-8. https://doi.org/10.1001/jamainternmed.2017.3947.

17. Wakeman SE, Metlay JP, Chang Y, Herman GE, Rigotti NA. Inpatient addiction consultation for hospitalized patients increases post-discharge abstinence and reduces addiction severity. J Gen Intern Med. 2017;32(8): 909-16. https://doi.org/10.1007/s11606-017-4077-z.

18. Priest KC, McCarty D. The role of the hospital in the 21 st century opioid overdose epidemic: the addiction medicine consult service. J Addict Med. 2019;13(2):104-12. https://doi.org/10.1097/ADM. 0000000000000496

19. Wakeman SE, Kane M, Powell E, Howard S, Shaw C, Regan S. Impact of inpatient addiction consultation on hospital readmission. J Gen Intern Med. 2020;36(7):1-3. https://doi.org/10.1007/s11606-020-05966-0.

20. Marks LR, Munigala S, Warren DK, Liang SY, Schwarz ES, Durkin MJ. Addiction medicine consultations reduce readmission rates for patients with serious infections from opioid use disorder. Clin Infect Dis. 2019 May 17; 68(11):1935-7. https://doi.org/10.1093/cid/ciy924. 
21. Snow RL, Simon RE, Jack HE, Oller D, Kehoe L, Wakeman SE. Patient experiences with a transitional, low-threshold clinic for the treatment of substance use disorder: a qualitative study of a bridge clinic. J Subst Abuse Treat. 2019;107:1-7. https://doi.org/10.1016/j.jsat.2019.09.003.

22. Wurcel AG, Anderson JE, Chui KK, Skinner S, Knox TA, Snydman DR, et al. Increasing infectious endocarditis admissions among young people who inject drugs. In: Open forum infectious diseases. Oxford University Press; 2016. p. ofw157.

23. Fleischauer AT, Ruhl L, Rhea S, Barnes E. Hospitalizations for endocarditis and associated health care costs among persons with diagnosed drug dependence-North Carolina, 2010-2015. MMWR Morb Mortal Wkly Rep. 2017;66(22):569-73. https://doi.org/10.15585/mmwr.mm6622a1.

24. Tookes H, Diaz C, Li H, Khalid R, Doblecki-Lewis S. A cost analysis of hospitalizations for infections related to injection drug use at a county safety-net hospital in Miami. Florida. PloS One. 2015;10(6):e0129360. https:// doi.org/10.1371/journal.pone.0129360.

25. Volkow ND, Koob GF, McLellan AT. Neurobiologic advances from the brain disease model of addiction. N Engl J Med. 2016;374(4):363-71. https://doi. org/10.1056/NEJMra1511480.

26. Chan A-W, Tetzlaff JM, Altman DG, Laupacis A, Gøtzsche PC, Krleža-Jerić K, et al. SPIRIT 2013 statement: defining standard protocol items for clinical trials. Ann Intern Med. 2013;158(3):200-7. https://doi.org/10.7326/0003-481 9-158-3-201302050-00583.

27. Harris PA, Taylor R, Thielke R, Payne J, Gonzalez N, Conde JG. Research electronic data capture (REDCap) - a metadata-driven methodology and workflow process for providing translational research informatics support. J Biomed Inform. 2009;42(2):377-81. https://doi.org/10.1016/j.jbi.2008.08.010.

28. Blais MA, Lenderking WR, Baer L, deLorell A, Peets K, Leahy L, et al. Development and initial validation of a brief mental health outcome measure. J Pers Assess. 1999;73(3):359-73. https://doi.org/10.1207/S1532 7752JPA7303_5.

29. Laux JM, Ahern B. Concurrent validity of the Schwartz Outcome Scale with a chemically dependent population. J Addict Offender Couns. 2003;24(1):210. https://doi.org/10.1002/j.2161-1874.2003.tb00177.x.

30. Haggerty G, Aflatoon K, Ghosh K, Babalola R, Ahmed Z, Alois A. Construct validity of the Schwartz Outcome Scale: validation using a 28-day inpatient chemical rehabilitation patient sample. J Nerv Ment Dis. 2012;200(4):343-8. https://doi.org/10.1097/NMD.0b013e31824cc210.

31. Suzuki J, Johnson J, Montgomery M, Hayden M, Price C. Outpatient parenteral antimicrobial therapy among people who inject drugs: a review of the literature. In: Open forum infectious diseases. US: Oxford University Press; 2018. p. ofy 194.

32. Eaton EF, Mathews RE, Lane PS, Paddock CS, Rodriguez JM, Taylor BB, et al. A 9-point risk assessment for patients who inject drugs and require intravenous antibiotics: focusing inpatient resources on patients at greatest risk of ongoing drug use. Clin Infect Dis. 2019;68(6):1041-3. https://doi.org/1 $0.1093 /$ cid/ciy722.

33. Williams AR, Nunes EV, Bisaga A, Pincus HA, Johnson KA, Campbell AN, et al. Developing an opioid use disorder treatment cascade: a review of quality measures. J Subst Abuse Treat. 2018;91:57-68. https://doi.org/10.1016/j.jsat.2 018.06.001.

34. Bray JW, Aden B, Eggman AA, Hellerstein L, Wittenberg E, Nosyk B, et al. Quality of life as an outcome of opioid use disorder treatment: a systematic review. J Subst Abuse Treat. 2017;76:88-93. https://doi.org/10.1016/j.jsat.201 7.01.019.

35. Patsopoulos NA. A pragmatic view on pragmatic trials. Dialogues Clin Neurosci. 2011;13(2):217-24. https://doi.org/10.31887/DCNS.2011.13.2/npa tsopoulos.

\section{Publisher's Note}

Springer Nature remains neutral with regard to jurisdictional claims in published maps and institutional affiliations.

Ready to submit your research? Choose BMC and benefit from:

- fast, convenient online submission

- thorough peer review by experienced researchers in your field

- rapid publication on acceptance

- support for research data, including large and complex data types

- gold Open Access which fosters wider collaboration and increased citations

- maximum visibility for your research: over $100 \mathrm{M}$ website views per year

At BMC, research is always in progress.

Learn more biomedcentral.com/submissions 